\title{
Review
}

\section{Progress in Research on the Mechanism and Treatment of Post- stroke Infection}

\author{
Hongtao Zhang \\ Department of Internal Medicine, Qingzhou Municipal Hospital, Qingzhou China
}

\author{
Keywords \\ stroke; infection; immune; inflammation; review \\ Correspondence \\ Hongtao Zhang, \\ E-mail: zhanght@sina.com
}

DOI: 10.1515/ii-2017-0134

\begin{abstract}
Post-stroke infection hinders the recovery of stroke patients and can even cause death. The main mechanism of post-stroke infection is related with the post-stroke center, the activation of the peripheral immune system, and the release of inflammatory factors caused by the lesion area and pathophysiological changes in the body. Therefore, elucidating the body's abnormal immune inflammatory responses after stroke is crucial for the prevention, treatment, and alleviation of post-stroke infection.
\end{abstract}

Post-stroke secondary injury is an important factor that affects the prognosis of patients. Post-stroke infection is the most common and deadly complication of stroke. In 2006, Vargas et al. ${ }^{[1]}$ proposed the concept of stroke-associated infection (SAI), and that pneumonia, urinary tract infections, and sepsis are the main factors that hinder the rehabilitation of patients and even cause death. The immune inflammatory response induced after stroke is an independent risk factor for the occurrence of SAI in patients. The main presentations of SAI are the activation of dendritic cells, macrophages, other antigen-presenting cells, and $\mathrm{T}$ and $\mathrm{B}$ lymphocytes, as well as the imbalanced secretion of pro-inflammatory/antiinflammatory factors. This paper provides a review of the pathophysiological changes associated with post-stroke infections to provide new ideas for the prevention of poststroke infection.

\section{Relevant Pathways of Post-stroke Infection}

Janus Protein Tyrosine Kinase/Signal Transducer and Activator of Transcription Pathway

The Janus protein tyrosine kinase (JAK)/signal transducer and activator of transcription (STAT) signaling pathway has become widely studied in recent years. Recent studies have shown that the JAK2/STAT pathway is an important signal transduction pathway in immune inflammatory response after cerebral ischemia. Although this pathway is usually inactive, after brain injury, T-lymphocyte surface cytokine receptor intracellular region JAK are activated to catalyze the phosphorylation of STAT with the SH2 structural domain. Relevant cytokine genes, such as IL-6R and IL$4 \mathrm{R}$, are activated to produce an immune response cascade reaction ${ }^{[3]}$. The activation of the JAK/STAT pathway could lead to the activation of glial cells and $\mathrm{T}$ and $\mathrm{B}$ lymphocytes, as well as upregulate the expression of IFN- $\gamma$, IL- 6 , and other immune inflammatory factors ${ }^{[4]}$. Current studies remain focused on the roles of the JAK/STAT pathway in cardiovascular diseases and tumors. The immune response produced by the pathway after stroke still requires further study.

\section{Fas/Fasl pathway}

Fas is widely expressed in the surfaces of cell membranes and is also known as apoptotic protein-1 or CD95 molecule. Fas belongs to the tumor necrosis factor/nerve growth factor receptor family. The role of Fas in immune adjustment in the lung, liver, and other organs has been widely studied. Recent studies ${ }^{[5]}$ have found that the Fasligand (Fasl), which extensively exists in microglia, astrocytes, lymphocytes, natural killer cells, monocytes, and other immune cells, is highly expressed after brain injury and produces immune inflammatory responses. At the same time, Fas1 and Fas combine via cross-linking to form a trimer or multimer, 
activating the cysteine protease (Caspase) family, which subsequently leads to the apoptosis of $\mathrm{T}$ lymphocytes and other immune cells, further intensifying brain injury. Related studies have shown that the activation of the CD95 signaling pathway in the brain after brain injury exerts protective effects by stimulating the regeneration of neural stem cells and promoting the recovery of neurological function ${ }^{[6]}$. Niu et al. ${ }^{[7]}$ found that in cerebral ischemia gld mice with Fasl gene mutation, FAS can improve the symptoms of neurological function deficit by inhibiting the infiltration of peripheral and main center inflammatory cells.

\section{Guanine Nucleotide Exchange Factor/Small Molecule G Protein Rho Pathway}

The guanine nucleotide exchange factor (GEF)/small molecule G protein Rho (Ras homologous, Rho) pathway also has a regulatory role in the diseases of the central nervous system ${ }^{[8]}$. In addition, GEF activation has multiple effects on the incidence of infection after cerebral ischemia. Rho belongs to the Ras superfamily, which includes the Rho (RhoA, RhoB, and RhoC), Rac, and Cdc42 subfamilies. Upstream GEFs bind to $G$ protein-coupled receptors (GPCRs) by releasing various growth factors, chemokines, TNF- $\alpha$, and lipopolysaccharides to activate RhoA and downstream Rho kinases; these interactions cause the dysfunction of brain microvascular endotheial cells (BMECs). Given the close relationship of BMECs and the blood-brain barrier (BBB) with integrity, the dysfunction of BMECs further causes structural and functional damage to the $\mathrm{BBB}{ }^{[9]}$ and increases permeability. In turn, the increased permeability of the $\mathrm{BBB}$ enables immune response inflammatory factors to permeate the $\mathrm{BBB}$ into the cerebral ischemic area after stroke, thus aggravating brain damage.

\section{Mechanisms of Post-stroke Infection}

\section{Central immune regulation}

Astrocytes, microglia, and neurons in injured brain tissues could activate inflammatory cells after stroke. The secretion of Toll-like receptors (TLR) increases to further activate downstream nuclear factor kappa-B (NF$\kappa B)$, mitogen-activated proteinkinase (MAPK), and other relevant inflammatory pathways to promote the infiltration of inflammatory factors, such as IL-4, IL-6, IFN- $\gamma$, and IL-1 $\beta$, in the nidus area of the cerebral ischemia. Meanwhile, the secretion of neutrophils, monocytes, and macrophages increases, thus up-regulating the expression of major histocompatibility complex I Class I (MHC I) and major histocompatibility complex class II (MHC II) and aggravating brain injury in the ischemic area.

\section{Peripheral immune regulation mechanism}

Changes in the peripheral immune system and the functional recovery of the injured brain area after stroke are also closely interrelated. The liver, spleen, lung, and other peripheral immune organs mainly regulate peripheral immune function. After brain damage, the central inflammatory response occurs in the hypothalamic paraventricular nucleus. This response releases glucocorticoid or adrenal cortex hormones through the adrenal cortex axis. The central inflammatory response can also occur through the sympathetic nervous system to directly activate peripheral T lymphocytes, macrophages, other immune cells, and chemokines ${ }^{[10]}$. These factors ultimately permeate the damaged BBB via blood circulation or osmotic effect to reach the lesion area, thus increasing brain damage.

A study by Stewart et al. ${ }^{[11]}$ showed that after cerebral ischemia, the $\alpha$-and $\beta$-adrenergic receptors of the spleen, a major reservoir of peripheral immunity, combine with catecholamines released by the center. This interaction results in the secretion of spleen neutrophils, macrophages, $\mathrm{C}$-reactive proteins, chemokines, and other secreted factors. NK, B, and $\mathrm{T}$ cells then undergo apoptosis. Th1 transforms into the Th2 subtype and the secretion of inflammatory factors increases. At the same time, inflammatory markers enter the damaged BBB with blood circulation and reach the cerebral ischemia area to increase brain damage. Wong et al. ${ }^{[12]}$ compared the molecular responses of wild-type mice with those of mice with liver iNKT cell deficiency after stroke. They found that liver iNKT cells can inhibit the release of norepinephrine receptor neurotransmitters and decrease inflammatory responses after stroke.

\section{Factors Related to Post-stroke Infection}

\section{Pathophysiological factors \\ Changes in immune organs}

The body's immune organs are the bone marrow, thymus, central immune organs, the spleen, liver, lymph nodes, and peripheral immune organs. Drechsler et al. ${ }^{[13]}$ pointed out that in mice with Apoe gene knockout, the bone marrow 
promotes the release of glial cells, bone marrow cells, and other inflammatory markers, thus increasing the count of the peripheral spleen and blood myeloid cells to induce and aggravate infection. The thymus and spleen indexes are objective indexes for the evaluation of immune function in animal experiments. After stroke, the immune stress response can cause the apoptosis of peripheral immune organ cells, causing the shrinkage of the spleen, thymus, and other immune organs, as well as the decline of immune functions.

\section{Dynamic changes in immune cells/cytokines}

In an induced cerebral ischemia animal model, post-stroke infection occurs rapidly given the rapid activation of the body's immune response. The inhibition of immune function can be detected several hours after cerebral ischemia and can last for several weeks ${ }^{[14]}$. However, the timing of post-stroke immunosuppression varies in different parts of the body.

$\mathrm{Gu}$ et al. ${ }^{[15]}$ showed that 3 days after cerebral ischemia, the numbers of CD $4+\mathrm{T}$ and CD $8+\mathrm{T}$ lymphocytes significantly increased in the ischemic hemispheres of a rat model compared with that in the sham operation group. Leilei et al. ${ }^{[16]}$ observed that the ratio of CD $4+/ \mathrm{CD} 8+\mathrm{T}$ lymphocytes in the peripheral blood of a cerebral ischemiareperfusion mouse model $24 \mathrm{~h}$ after stroke was higher than that in the sham operation group. The ratio of CD4 +/CD8 + T lymphocytes were not different $6 \mathrm{~h}$ and $96 \mathrm{~h}$ after stroke. However, at $96 \mathrm{~h}$ after stroke, the ratios of CD4 $+/ \mathrm{CD} 8+\mathrm{T}$ lymphocytes decreased and the expression of proinflammatory factors, such as TNF- $\alpha$ and IFN- $\gamma$, were inhibited in the spleen. At 6 and $24 \mathrm{~h}$ after stroke, the expressions of pro-inflammatory and anti-inflammatory factors in the spleen and serum increased, whereas that of serum IFN- $\gamma$ did not change $6 \mathrm{~h}$ after stroke. Liesz et al. ${ }^{[17]}$ used the cerebral ischemia mouse model to show that lymphocyte counts decreased in the spleen, lymph nodes, and thymus 24 h, 3 days, and 7 days after stroke, whereas TNF- $\alpha$ and IFN- $\gamma$ secretion increased. By comparing the levels of the inflammatory cytokine CXCL-1 in the brain and peripheral immune organs, Chapman et al. ${ }^{[18]}$ found differences in central and peripheral inflammatory response: at $24 \mathrm{~h}$ after stroke, CXCL-1 in the serum, liver, and lung returned to normal but continued to increase in the brain.

\section{Other factors}

Approximately $30 \%$ of stroke patients with the mass propagation of oral pathogens and malnutrition caused by swallowing dysfunction ${ }^{[19]}$ need enteral nutrition supply [2]. These factors easily induce infection. At the same time, co-occurrence with other diseases (such as diabetes and cardiovascular disease, etc.) or post-stroke limb dysfunction increases the incidence of infection.

\section{Treatment Strategies for Post-stroke Infection}

\section{Drug treatment}

\section{Antibiotic treatment}

The central and peripheral immune systems undergo changes several hours after stroke. Preventive anti-infective treatment is usually needed to prevent stroke. A multi-center and open clinical experiment (Preventive Antibiotics in Stroke Study, PASS) on 2550 stroke patients found that the incidence of post-stroke infection significantly decreased 3 months after using cephalosporins, which are third-generation antibiotics, for $4 \mathrm{~d}^{[20]}$. However, a study pointed out that ${ }^{[21]}$ although cephalosporin can prevent post-stroke infection, the therapeutic efficacy of the drug on different infections, drug resistance caused by long-term use, and other problems should be studied further. Studies have shown that ${ }^{[22]} 3-$ $4 \mathrm{~d}$ after stroke, treatment with penicillin and sulbacam, a $\beta$-receptor retardant, can decrease the incidence of poststroke infection. The anti-infective effect of the quinolone antibiotic moxifloxacin is superior to that of levofloxacin. However, although some drugs have anti-infective effects, they may also increase brain damage by exerting toxic effects on the central nervous system.

\section{Other therapy drugs}

Chen Shuzeng et al. ${ }^{[23]}$ pointed out that preventive treatment with the lipid-lowering drug fluvastatin can down-regulate TNF- $\alpha$ expression and up-regulate IL-10 expression in the brain tissue of rats with cerebral ischemia. These effects decrease post-stroke inflammatory immune responses to protect the brain. However, studies have shown that the early use of statins increases the level of serum interleukin-1 receptor antagonists and increases the risk of infection in stroke patients. Some scholars ${ }^{[25]}$ have reported that 7 days after levodopa treatment, the up-regulation of peripheral blood CD3 + CD4 + T lymphocyte levels can improve immunosuppression status after stroke. Given the difference in the gender and age of stoke patients, estrogen may exert neuroprotective effects. For example, in a male cerebral 
ischemia model, estrogen inhibits immune inflammatory response after stroke. However, estrogen may increase the incidence of stroke in menopausal female models ${ }^{[26]}$. As an immunomodulator, Graim can reduce the secretion of proinflammatory factors after stroke but does not improve the volume of post-stroke cerebral infarction and neurological deficits ${ }^{[27]}$.

\section{Peripheral immunotherapy}

To regulate post-stroke peripheral immune function, Yan et al. ${ }^{[28]}$ found that $24 \mathrm{~h}$ after stroke, the transplantation of human umbilical cord blood stem cells (HUCBC) can repair $\mathrm{BBB}$ damage caused by cerebral ischemia and reduce inflammation factor release, resulting in neuroprotective effects. Zhang et al. ${ }^{[29]}$ found that performing splenectomy 2 weeks before permanent cerebral ischemia decreases the number of T cells, neutrophils, and macrophages in the brain tissue of rats and decreases the release of proinflammatory cytokines, such as IL $-1 \beta$ and other factors, and thus increasing the secretion of anti-inflammatory factor IL-10. Although splenectomy can decrease the incidence of poststroke infection to a certain extent, its effects on the normal physiological function of the spleen still require further study.

\section{Traditional Chinese medicine}

The traditional Chinese medicines Buyang Huanwu decoction ${ }^{[30]}$, an astragalus-based decoction, and Shenlong decoction, a ginseng- and polygonum-multiflorumbased decoction ${ }^{[31]}$, can significantly improve post-stroke immunosuppression to decrease the incidence of post-stroke infection. The results of animal experiments have confirmed that ${ }^{[32]}$ acupuncture at the Baihui and Zusanli acupoints are beneficial for the recovery of brain function in rats with cerebral ischemia. Acupuncture at these acupoints also down-regulates the expression of inflammatory markers, such as IL-1 $\beta$ and IL-6, thereby alleviating the inflammatory immune response mediated by these markers and attenuating cerebral ischemia-reperfusion injury to exert protective effects on brain tissue.

\section{Other methods}

Some natural food and plant ingredients exert effects that decrease inflammatory responses and the likelihood of infection after stroke. Qiao et al. ${ }^{[33]}$ reported that luteolin extracted from natural plants could down-regulate the levels of Toll-like receptor (TLR)-4, TLR5, NF- $\kappa$ B, and other inflammatory factors in a rat model of cerebral ischemia, thus decreasing inflammatory response and protecting the brain. Wang et al. ${ }^{[34]}$ found that after 3 days of continuous lavege with plant-derived shikonin, cerebral ischemia-reperfusion mice exhibited the decreased expression of inflammatory markers, such as TLR4, TNF- $\alpha$, and phosphorylated p38 mitogen-activated protein kinases. These effects inhibit $\mathrm{NF}-\kappa \mathrm{B}$ nuclear translocation, repair the $\mathrm{BBB}$, and exert neuroprotective effects. In addition, the high carnosine content of beef and chicken has a certain immunoregulatory function in cerebral ischemia, thereby reducing the incidence of post-stroke infection to protect the brain ${ }^{[35]}$.

\section{Conclusion}

Methods for the prevention of post-stroke infection have mainly focused on the regulation of immune function and the standardization of invasive surgery. Elucidating poststroke pathophysiological functions is crucial to decrease the incidence of infection in stroke patients. Exploring the dynamic changes in central and peripheral immune regulation after stroke could provide a specific target for combination therapy. The future treatment of stroke patients with infection should be based on molecular markers, immune cells, and immunoinflammatory pathways. To improve the recovery of the patient from post-stroke infection, clinicians should provide customized and comprehensive treatment via medication, diet, and physical therapy.

\section{Declarations}

\section{Acknowledgements}

No.

\section{Competing interests}

The author declares that he has no competing interest.

\section{Authors' contributions}

HT Zhang made the literature analysis and wrote, discussed and revised the manuscript of this review.

\section{References}

1 Vargas M, Horcajada JP, Obach V, et al. Clinical consequences of 
infection in patients with acute stroke: is it prime time for further antibiotic trials? Stroke, 2006, 37(2): 461-465.

2 Brogan E, Langdon C, Brookes K, et al. Can't swallow, can't transfer, can' $\mathrm{t}$ toilet: factors predicting infections in the first week post stroke. J Clin Neurosci, 2015, 22(1): 92-97.

3 George R, Stark James E, Darnell Jr. The JAK/STAT pathway at twenty. Immunity, 2012, 36(4): 503-514.

4 Eufemi M, Cocchiola R, Romaniello D, et al. Acetylation and phosphorylation of STAT3 are involved in the responsiveness of microglia to beta amyloid. Neurochem Int, 2015, 81: 48-56.

5 Bonnaure G, Néron S. N-acetyl cysteine regulates the phosphorylation of JAK proteins following CD40-activation of human memory B cells. Mol Immunol, 2014, 62(1): 209-218.

6 Corsini NS, Sancho-Martinez I, Laudenklos S, et al. The death receptor CD95 activates adult neural stem cells for working memory formation and brain repair. Cell Stem Cell, 2009, 5(2): 178-190.

7 Niu FN, Zhang X, Hu XM, et al. Targeted mutation of Fas ligand gene attenuates brain inflammation in experimental stroke. Brain Behav Immun, 2012, 26(1): 61-71.

8 DeGeer J, Lamarche-Vane N. Rho GTPases in neurodegeneration diseases. Exp Cell Res, 2013, 319(15): 2 384-2 394.

9 Wang C, Wang C, Dong H, et al. Immune-related GTPase Irgm 1 exacerbates experimental auto-immune encephalomyelitis by promoting the disruption of blood-brain barrier and bloodcerebrospinal fluid barrier. Mol Immunol, 2013, 53(1/2): 43-51.

10 Mackroth MS, Malhotra I, Mungai P, et al. Human cord blood CD4 + CD25hi regulatory $\mathrm{T}$ cells suppress prenatally acquired $\mathrm{T}$ cell responses to Plasmodium falciparum antigens. J Immunol, 2011, 186(5): 2780-2791.

11 Stewart IB, Mckenzie DC. The human spleen during physiological stress. Sports Med, 2002, 32(6): 361-369.

12 Wong $\mathrm{CH}$, Jenne CN, Lee WY, et al. Functional innervation of hepatic iNKT cells is immunosuppressive following stroke. Science, 2011, 334(652): 101-105.

13 Drechsler M, Megens RT, van Zandvoort M, et al. Hyperlipidemiatriggered neutrophilia promotes early atherosclerosis. Circulation, 2010, 122(18): $1837-1845$.

14 Westendorp WF, Nederkoorn PJ, Vermeij JD, et al. Post-stroke infection: a systematic review and meta-analysis. BMC Neurol, 2011, 11: 110.

15 Gu L, Xiong X, Wei D, et al. T cells contribute to stroke-induced lymphopenia in rats. PLoS One, 2013, 8(3): e59 602.

16 Chang L, Chen Y, Li J, et al. Cocaine-and a mphetamine-regulated transcript modulates peripheral immunity and protects against brain injury in experimental stroke. Brain Behav Immun, 2011, 25(2): 260-269.

17 Liesz A, Hagmann S, Zschoche C, et al. The spectrum of systemic immune alterations after murine focal ischemia: immunodepression versus immunomodulation. Stroke, 2009, 40 (8):2 849-2 858.
18 Chapman KZ, Dale VQ Dénes A, et al. A rapid and transient peripheral inflammatory response precedes brain inflammation after experimental stroke. J Cereb Blood Flow Metab, 2009, 29(11): 1 764-1 768.

19 Hankey GJ. Nutrition and the risk of stroke. Lancet Neurol, 2012, 11(1): 66-81.

20 Westendorp WF, Vermeij JD, Zock E, et al. The Preventive Antibiotics in Stroke Study(PASS): a pragmatic randomised openlabel masked endpoint clinical trial. Lancet, 2015, 385(9977): 1519-1 526.

21 Meisel A. Preventive antibiotic therapy in stroke: PASSed away? Lancet, 2015, 385(9977): 1 486-1 487.

22 Schwarz S, Al-Shajlawi F, Sick C, et al. Effects of prophylactic antibiotic therapy with mezlocillin plus sulbactam on the incidence and height of fever after severe acute ischemic stroke: the Mannheim infection in stroke study(MISS). Stroke, 2008, 39(4): 1 220-1 227.

23 Chen Shuzeng, Wang Jiehua. Effects of Fluvastatin Pretreatment on Expression of TNF- $\alpha$ and IL-10 in Brain Tissue of Rats with Cerebral Ischemia-Reperfusion injury. Immunological Journal, 2012, 28 (4): 309-311, 316.

24 Becker K, Tanzi P, Kalil A, et al. Early statin use is associated with increased risk of infection after stroke. J Stroke Cerebrovasc Dis, 2013, 22(1): 66-71.

25 Kuric E, Ruscher K. Reversal of stroke induced lymphocytopenia by levodopa/benserazide treatment. J Neuroimmunol, 2014, 269(1-2): 94-97.

26 Ritzel RM, Capozzi LA, Mccullough LD. Sex, stroke, and inflammation: the potential for estrogen-mediated immunoprotection in stroke. Horm Behav, 2013, 63(2): 238-253.

27 Poittevin M, Deroide N, Azibani F, et al. Glatiramer Acetate administration does not reduce damage after cerebral ischemia in mice. J Neuroimmunol, 2013, 254(1-2): 55-62.

28 Yan T, Venkat $\mathrm{P}, \mathrm{Ye} X$, et al. HUCBCs increase angiopoietin 1 and induce neurorestorative effects after stroke in T1DM rats. CNS Neurosci Ther, 2014, 20(10): 935-944.

29 Zhang B, Men X, Lu Z, et al. Splenectomy protects experimental rats from cerebral damage after stroke due to anti-inflammatory effects. Chin Med J (Engl), 2013, 126 (12):2354-2 360.

30 Liu N, Peng K. Study on the Effect of Buyang Huanwu Decoction on Neuroendocrine and Immune Function in Stroke. Chinese Archives of Traditional Chinese Medicine, 2004, 22 (10): 1898-1900.

31 Chen L, Wang F, Sun Z, et al. Effects of Shenlong Decoction on Serum Inflammatory Factors in Rats with Cerebral Ischemia. Journal of Emergency in Traditional Chinese Medicine, 2013, 22 (2): 201-202, 213.

32 Effects of Acupuncture on Expression of IL-1 $\beta$ and IL- 6 in Peripheral Blood of Rats with Cerebral Ischemia-reperfusion Injury. Journal of Clinical Acupuncture and Moxibustion, 2013, 29 (1): 60-63.

33 Qiao H, Zhang X, Zhu C, et al. Luteolin downregulates TLR4, TLR5, NF- $\kappa B$ and p-p38MAPK expression, upregulates the p-ERK expression, 
and protects rat brains against focal ischemia. Brain Res, 2012, 1448 : 71-81.

34 Wang L, Li Z, Zhang X, et al. Protective effect of shikonin in experimental ischemic stroke: attenuated TLR4, p-p38MAPK, NF-kappa B, TNFalpha and MMP-9 expression, up-regulated claudin-5 expression, ameliorated BBB permeability. Neurochem Res, 2014, 39(1): 97-106.

35 Bae ON, Serfozo K, Baek SH, et al. Safety and efficacy evaluation of carnosine, an endogenous neuroprotective agent for ischemic stroke. Stroke, 2013, 44(1): 205-212. 